\section{Improved Global Robust Asymptotic Stability Criteria for Delayed Cellular Neural Networks}

\author{
Shengyuan Xu, James Lam, Daniel W. C. Ho, and Yun Zou
}

\begin{abstract}
This paper considers the problem of global robust stability analysis of delayed cellular neural networks (DCNNs) with norm-bounded parameter uncertainties. In terms of a linear matrix inequality, a new sufficient condition ensuring a nominal DCNN to have a unique equilibrium point which is globally asymptotically stable is proposed. This condition is shown to be a generalization and improvement over some previous criteria. Based on the stability result, a robust stability condition is developed, which contains an existing robust stability result as a special case. An example is provided to demonstrate the reduced conservativeness of the proposed results.
\end{abstract}

Index Terms-Cellular neural network, global asymptotic stability, linear matrix inequality, parameter uncertainty, robust stability, time delays.

\section{INTRODUCTION}

Cellular neural networks (CNNs), which were introduced in [8] and [9], have received much attention in the past years due to their extensive applications in signal processing, moving image processing, optimization, speed detection of moving objects, and other areas [8]. It has been shown that such applications rely on the existence of equilibrium points or of a unique equilibrium point and its qualitative properties of stability. For example, when a $\mathrm{CNN}$ is applied to image processing where the main function of CNNs is to transform an input image into a corresponding output image, it is then necessary that the CNN must be completely stable in the sense that every trajectory converges to an equilibrium point. Therefore, stability analysis of CNNs has been studied recently and a great number of results on this topic have been reported in the literature via various approaches.

In implementations of artificial neural networks, however, time delays may occur due to finite switching speeds of the amplifiers and communication time. Time delay is often a source of instability and poor performance of a CNN. Considering this, the problem of stability analysis of delayed cellular neural networks (DCNNs) has become one of the most active research areas and has attracted much attention during the past years [5]-[7]. For instance, a sufficient condition ensuring the uniqueness and global asymptotic stability of an equilibrium point of DCNNs was proposed in [5]; this was shown to be less conservative than some earlier asymptotic stability results in [3], [11], and [13]. Via a new approach, sufficient conditions for the uniqueness and global asymptotic stability for a DCNN was proposed in [12]. Very recently, a linear matrix inequality (LMI) approach was developed and a sufficient condition for global asymptotic stability of DCNNs was given in

Manuscript received August 19, 2004; revised February 3, 2005. This work was supported in part by HKU CRCG 10205878 , by the Program for New Century Excellent Talents in University, by the National Natural Science Foundation of China under Grants 60304001 and 60474078, by the Fok Ying Tung Education Foundation under Grant 91061, and by the Foundation for the Author of National Excellent Doctoral Dissertation of China under Grant 200240. This paper was recommended by Associate Editor P. S. Sastry.

S. Xu and Y. Zou are with the Department of Automation, Nanjing University of Science and Technology, Nanjing 210094, China (e-mail: syxu02@ yahoo.com.cn).

J. Lam is with the Department of Mechanical Engineering, University of Hong Kong, Hong Kong.

D. W. C. Ho is with the Department of Mathematics, City University of Hong Kong, Kowloon, Hong Kong.

Digital Object Identifier 10.1109/TSMCB.2005.851539
[14]; it was shown in [14] that the asymptotic stability results in [1], [5], [11] are special cases of that in [14]. When norm-bounded parameter uncertainties appear in a DCNN, a sufficient condition for robust stability was obtained in [15] via an LMI approach.

In this paper, we deal with the problem of robust stability analysis for DCNNs. The parameter uncertainties are assumed to be norm-bounded and appear in both the feedback and delayed feedback matrices. Firstly, a sufficient condition ensuring the uniqueness and global asymptotic stability of the equilibrium point for a nominal DCNN is developed. This condition is expressed in terms of an LMI, which can be checked numerically very efficiently by resorting to recently developed interior-point methods, and no tuning of parameters will be involved [4]. Based on the asymptotic stability result, a new global robust asymptotic stability condition is obtained in terms of an LMI. It is theoretically established that the developed stability result in this paper contains the earlier asymptotic stability results in [1], [2], [11], and [14] as special cases, while the derived robust stability condition contains that in [15] as a special case. A numerical example is provided to demonstrate the reduced conservatism of the proposed results in this paper.

Notation. Throughout this paper, for real symmetric matrices $X$ and $Y$, the notation $X \geq Y$ (respectively, $X>Y$ ) means that the matrix $X-Y$ is positive semi-definite (respectively, positive definite). The superscript " $T$ " represents the transpose. We use $\lambda_{\min }(\cdot)$ to denote the minimum eigenvalue of a real symmetric matrix. The notation $\|x\|$ denotes a vector norm defined by $\|x\|=\left(\sum_{i=1}^{n} x_{i}^{2}\right)^{1 / 2}$ when $x$ is a vector. Matrices, if not explicitly stated, are assumed to have compatible dimensions.

\section{PROBLEM Formulation}

Consider a $\mathrm{CNN}$ with a time delay and parameter uncertainties described by [15], as follows:

$$
\begin{aligned}
\dot{x}(t)= & -x(t)+(A+\Delta A) y(x(t)) \\
& +\left(A^{\tau}+\Delta A^{\tau}\right) y(x(t-\tau))+u
\end{aligned}
$$

or

$$
\begin{aligned}
\dot{x}_{i}(t)= & -x_{i}(t)+\sum_{j=1}^{n}\left(a_{i j}+\Delta a_{i j}\right) y_{j}\left(x_{j}(t)\right) \\
& +\sum_{j=1}^{n}\left(a_{i j}^{\tau}+\Delta a_{i j}^{\tau}\right) y_{j}\left(x_{j}(t-\tau)\right)+u_{i}, \quad i=1,2, \ldots, n
\end{aligned}
$$

where

$$
x(t)=\left[\begin{array}{llll}
x_{1}(t) & x_{2}(t) & \cdots & x_{n}(t)
\end{array}\right]^{T}
$$

is the state vector

$$
y(t)=\left[\begin{array}{llll}
y_{1}(t) & y_{2}(t) & \cdots & y_{n}(t)
\end{array}\right]^{T}
$$

is the output vector and

$$
u=\left[\begin{array}{llll}
u_{1} & u_{2} & \cdots & u_{n}
\end{array}\right]^{T}
$$

is the constant external input vector. $A=\left\{a_{i j}\right\}$ is the feedback matrix, $A^{\tau}=\left\{a_{i j}^{\tau}\right\}$ is the delayed feedback matrix, $\Delta A=\left\{\Delta a_{i j}\right\}$ and $\Delta A^{\tau}=\left\{\Delta a_{i j}^{\tau}\right\}$ represent the parameter uncertainties in the matrices $A$ and $A^{\tau}$, respectively. In this paper, we assume $\Delta A$ and $\Delta A^{\tau}$ are of the following form [15]:

$$
\Delta A=H F E, \quad \Delta A^{\tau}=H^{\tau} F^{\tau} E^{\tau}
$$

where

$$
F^{T} F \leq I, \quad\left(F^{\tau}\right)^{T} F^{\tau} \leq I
$$


$\Delta A$ and $\Delta A^{\tau}$ are said to be admissible if both (2) and (3) hold. In system (1), the scalar $\tau>0$ is the transmission delay, and $y_{i}$ are the activation functions, which are given by

$$
y_{i}\left(x_{i}\right)=\frac{1}{2}\left(\left|x_{i}+1\right|-\left|x_{i}-1\right|\right), \quad i=1,2, \ldots, n .
$$

Now, suppose that

$$
x^{*}=\left[\begin{array}{llll}
x_{1}^{*} & x_{2}^{*} & \cdots & x_{n}^{*}
\end{array}\right]^{T}
$$

is the equilibrium point of (1). Then, by setting

$$
z(t)=x(t)-x^{*}
$$

it can be shown that the equilibrium point $x^{*}$ of (1) can be shifted to the origin; in this case, system (1) is transformed to

$$
\dot{z}(t)=-z(t)+(A+\Delta A) \Phi(z(t))+\left(A^{\tau}+\Delta A^{\tau}\right) \Phi(z(t-\tau))
$$

where

$$
\begin{aligned}
z(t) & =\left[\begin{array}{llll}
z_{1}(t) & z_{2}(t) & \cdots & z_{n}(t)
\end{array}\right]^{T} \\
\Phi(z(t)) & =\left[\begin{array}{llll}
\Phi_{1}\left(z_{1}(t)\right) & \Phi_{2}\left(z_{2}(t)\right) & \cdots & \Phi_{n}\left(z_{n}(t)\right)
\end{array}\right]^{T} \\
\Phi_{i}\left(z_{i}(t)\right) & =y_{i}\left(z_{i}(t)+x_{i}^{*}\right)-y_{i}\left(x_{i}^{*}\right), \quad i=1,2, \ldots, n .
\end{aligned}
$$

It is easy to see that

$$
\Phi_{i}\left(z_{i}(t)\right)^{2} \leq z_{i}(t) \Phi_{i}\left(z_{i}(t)\right), \quad \Phi_{i}(0)=0, \quad i=1,2, \ldots, n .
$$

Then, the problem to be addressed in this paper is to develop improved global robust asymptotic stability conditions for the cellular neural network in (6) via an LMI approach.

\section{Global Stability and Robust Stability}

In order to develop improved robust stability conditions, we first consider the nominal DCNN of (6); that is

$$
\dot{z}(t)=-z(t)+A \Phi(z(t))+A^{\tau} \Phi(z(t-\tau)) .
$$

The following theorem provides a new asymptotic stability condition for the DCNN in (11).

Theorem 1: The origin of the DCNN in (11) is the unique equilibrium point and is globally asymptotically stable if there exist matrices $P>0, Q>0$ and two diagonal matrices $D>0$ and $S>0$ such that the following LMI holds:

$$
\left[\begin{array}{ccc}
-2 P & P A-D+S & P A^{\tau} \\
A^{T} P-D+S & D A+A^{T} D+Q-2 S & D A^{\tau} \\
\left(A^{\tau}\right)^{T} P & \left(A^{\tau}\right)^{T} D & -Q
\end{array}\right]<0 .
$$

Proof: Firstly, we show the uniqueness of the equilibrium point by contradiction. To this end, we let $\bar{z}$ be the equilibrium point of the delayed cellular neural network in (11), then we have

$$
-\bar{z}+\left(A+A^{\tau}\right) \Phi(\bar{z})=0 .
$$

Now, suppose $\bar{z} \neq 0$. Then, by (13), it is easy to see that

$$
\begin{aligned}
-2 \bar{z}^{T} P \bar{z}+2 \bar{z}^{T}\left[P\left(A+A^{\tau}\right)\right. & -D] \Phi(\bar{z}) \\
& +2 \Phi(\bar{z})^{T} D\left(A+A^{\tau}\right) \Phi(\bar{z})=0 .
\end{aligned}
$$

Noting that $S>0$ is diagonal and using (10), we have

$$
\Phi(\bar{z})^{T} S \Phi(\bar{z}) \leq \bar{z}^{T} S \Phi(\bar{z}) .
$$

This, together with (14), gives

$$
\begin{aligned}
- & 2 \bar{z}^{T} P \bar{z}+2 \bar{z}^{T}\left[P\left(A+A^{\tau}\right)-D+S\right] \Phi(\bar{z}) \\
& -\Phi(\bar{z})^{T}\left[2 S-D\left(A+A^{\tau}\right)\right. \\
& \left.-\left(A+A^{\tau}\right)^{T} D\right] \Phi(\bar{z}) \geq 0 .
\end{aligned}
$$

On the other hand, pre- and post-multiplying (12) by

$$
\left[\begin{array}{lll}
I & 0 & 0 \\
0 & I & I
\end{array}\right]
$$

and its transpose, respectively, we can deduce

$$
2 S-D\left(A+A^{\tau}\right)-\left(A+A^{\tau}\right)^{T} D>0,
$$

and

$$
\begin{gathered}
-2 P+\left[P\left(A+A^{\tau}\right)-D+S\right]\left[2 S-D\left(A+A^{\tau}\right)\right. \\
\left.-\left(A+A^{\tau}\right)^{T} D\right]^{-1}\left[P\left(A+A^{\tau}\right)-D+S\right]^{T}<0 .
\end{gathered}
$$

By (16) and Lemma 1 in [16], it can be shown that

$$
\begin{aligned}
2 \bar{z}^{T}[ & \left.P\left(A+A^{\tau}\right)-D+S\right] \Phi(\bar{z})-\Phi(\bar{z})^{T} \\
\times & {\left[2 S-D\left(A+A^{\tau}\right)-\left(A+A^{\tau}\right)^{T} D\right] \Phi(\bar{z}) } \\
\leq & \bar{z}^{T}\left[P\left(A+A^{\tau}\right)-D+S\right]\left[2 S-D\left(A+A^{\tau}\right)\right. \\
& \left.\quad-\left(A+A^{\tau}\right)^{T} D\right]^{-1}\left[P\left(A+A^{\tau}\right)-D+S\right]^{T} \bar{z} .
\end{aligned}
$$

Then, it follows from (15) and (18) that

$$
\begin{aligned}
\bar{z}^{T}\left(-2 P+\left[P\left(A+A^{\tau}\right)-D+S\right]\left[2 S-D\left(A+A^{\tau}\right)\right.\right. \\
\left.\left.-\left(A+A^{\tau}\right)^{T} D\right]^{-1}\left[P\left(A+A^{\tau}\right)-D+S\right]^{T}\right) \bar{z} \geq 0 .
\end{aligned}
$$

This contradicts (17). The contradiction implies that $\bar{z}=0$. That is, the origin of the delayed cellular neural network in (11) is the unique equilibrium point. In the following, we shall establish the global asymptotic stability of the unique equilibrium point of (11). To this end, we denote

$$
D=\operatorname{diag}\left(d_{1}, d_{2}, \ldots, d_{n}\right),
$$

and choose a Lyapunov-Krasovskii functional candidate for system (11) as

$$
\begin{aligned}
V\left(z_{t}, t\right)=z(t)^{T} P z(t)+2 & \sum_{i=1}^{n} d_{i} \int_{0}^{z_{i}(t)} \Phi_{i}(\alpha) d \alpha \\
& +\int_{t-\tau}^{t} \Phi(z(\alpha))^{T} Q \Phi(z(\alpha)) d \alpha
\end{aligned}
$$

where

$$
z_{t}=z(t+\theta), \quad-\tau \leq \theta \leq 0 .
$$

Then, the time-derivative of $V\left(z_{t}, t\right)$ along the solution of (11) gives

$$
\begin{aligned}
\dot{V}\left(z_{t}, t\right)= & -2 z(t)^{T} P z(t)+2 z(t)^{T}(P A-D) \Phi(z(t)) \\
& +2 z(t)^{T} P A^{\tau} \Phi(z(t-\tau)) \\
& +\Phi(z(t))^{T}\left(D A+A^{T} D+Q\right) \Phi(z(t)) \\
& +2 \Phi(z(t))^{T} D A^{\tau} \Phi(z(t-\tau)) \\
& -\Phi(z(t-\tau))^{T} Q \Phi(z(t-\tau)) .
\end{aligned}
$$

By (10), we obtain

$$
\Phi(z(t))^{T} S[z(t)-\Phi(z(t))] \geq 0 .
$$

Therefore

$$
\dot{V}\left(z_{t}, t\right) \leq \xi(t)^{T} \Lambda \xi(t)
$$

where

$$
\xi(t)=\left[z(t)^{T} \quad \Phi(z(t))^{T} \quad \Phi(z(t-\tau))^{T}\right]^{T},
$$

and

$$
\Lambda=\left[\begin{array}{ccc}
-2 P & P A-D+S & P A^{\tau} \\
A^{T} P-D+S & D A+A^{T} D+Q-2 S & D A^{\tau} \\
\left(A^{\tau}\right)^{T} P & \left(A^{\tau}\right)^{T} D & -Q
\end{array}\right]
$$


Then, by (12) and (22), it can be seen that

$$
\dot{V}\left(z_{t}, t\right) \leq-a\|z(t)\|^{2},
$$

where $a=\lambda_{\min }(-\Lambda)>0$. Finally, by [10], it follows from (23) that the DCNN in (11) is globally asymptotically stable. This completes the proof.

Remark 1: Theorem 1 provides a new sufficient condition for the global asymptotic stability of the DCNN in (11) in terms of an LMI. This LMI condition can be checked numerically very efficiently by using the interior-point algorithms which have been developed recently in solving LMIs [4].

Now, we are in a position to provide the robust asymptotic stability condition for the uncertain DCNN in (6).

Theorem 2: The origin of the uncertain DCNN in (6) is the unique equilibrium point and is globally asymptotically stable for all admissible uncertainties if there exist matrices $P>0, Q>0$, two diagonal matrices $D>0, S>0$, and scalars $\epsilon_{1}>0$ and $\epsilon_{2}>0$ such that the LMI shown in (24), at the bottom of the page, holds.

Proof: Noting (2) and (3), and using Lemma 1 in [16], we have

$$
\left[\begin{array}{ccc}
0 & P \Delta A & 0 \\
\Delta A^{T} P & D \Delta A+\Delta A^{T} D & 0 \\
0 & 0 & 0
\end{array}\right] \leq \epsilon_{1}^{-1} \Upsilon_{1} \Upsilon_{1}^{T}+\epsilon_{1} \Upsilon_{2}^{T} \Upsilon_{2}
$$

$$
\left[\begin{array}{ccc}
\text { and } & \\
0 & 0 & P \Delta A^{\tau} \\
0 & 0 & D \Delta A^{\tau} \\
\left(\Delta A^{\tau}\right)^{T} P & \left(\Delta A^{\tau}\right)^{T} D & 0
\end{array}\right] \leq \epsilon_{2}^{-1} \Xi_{1} \Xi_{1}^{T}+\epsilon_{2} \Xi_{2}^{T} \Xi_{2}
$$

where

$$
\begin{aligned}
& \Upsilon_{1}=\left[\begin{array}{lll}
H^{T} P & H^{T} D & 0
\end{array}\right]^{T}, \quad \Upsilon_{2}=\left[\begin{array}{lll}
0 & E & 0
\end{array}\right], \\
& \Xi_{1}=\left[\begin{array}{llll}
\left(H^{\tau}\right)^{T} P & \left(H^{\tau}\right)^{T} D & 0
\end{array}\right]^{T}, \quad \Xi_{2}=\left[\begin{array}{lll}
0 & 0 & E^{\tau}
\end{array}\right] .
\end{aligned}
$$

Now, applying Schur complements to (24) and using (25) and (26), we have the second equation shown at the bottom of the page. Finally, by this inequality and Theorem 1 , the desired result follows immediately.

\section{COMPARISON With PREVIOUS CRITERIA}

In the literature, a great number of asymptotic stability and robust stability results for the DCNN in (11) have been reported. In this section, we will show that the stability conditions in [1], [2], [11], and [14] are the special cases of our results in Theorem 1, while the robust stability condition in [15] is a special case of our result in Theorem 2. To this end, we first restate the results in [2], [14], and [15] as follows.

Theorem 3: [14]: The origin of the DCNN in (11) is the unique equilibrium point and is globally asymptotically stable if there exist matrices $P>0, Q>0$ and a diagonal matrix $D>0$ such that the following LMI holds:

$$
\left[\begin{array}{ccc}
2 P & -P A & -P A^{\tau} \\
-A^{T} P & 2 D-D A-A^{T} D-Q & -D A^{\tau} \\
-\left(A^{\tau}\right)^{T} P & -\left(A^{\tau}\right)^{T} D & Q
\end{array}\right]>0 .
$$

Theorem 4: [2]: The origin of the DCNN in (11) is the unique equilibrium point and is globally asymptotically stable if there exist a matrix $K>0$ and a diagonal matrix $P>0$ such that the following matrix inequalities hold:

$$
\begin{array}{r}
P A+A^{T} P+K<0 \\
-2 P-K+I+P A^{\tau}\left(A^{\tau}\right)^{T} P \leq 0 .
\end{array}
$$

Theorem 5: [15]: The origin of the uncertain DCNN in (6) is the unique equilibrium point and is globally asymptotically stable for all admissible uncertainties if there exist a diagonal matrix $D>0$ and scalars $\epsilon_{1}>0, \epsilon_{2}>0$ such that the LMI, shown in (30) at the bottom of the page, holds.

Now, we have the following results.

Theorem 6: Suppose that there exist matrices $P>0, Q>0$ and a diagonal matrix $D>0$ such that the LMI in (27) holds, then there exist matrices $\tilde{P}>0, \tilde{Q}>0$ and two diagonal matrices $\tilde{D}>0$ and $\tilde{S}>0$ satisfying the LMI in (12); that is

$$
\left[\begin{array}{ccc}
-2 \tilde{P} & \tilde{P} A-\tilde{D}+\tilde{S} & \tilde{P} A^{\tau} \\
A^{T} \tilde{P}-\tilde{D}+\tilde{S} & \tilde{D} A+A^{T} \tilde{D}+\tilde{Q}-2 \tilde{S} & \tilde{D} A^{\tau} \\
\left(A^{\tau}\right)^{T} \tilde{P} & \left(A^{\tau}\right)^{T} \tilde{D} & -\tilde{Q}
\end{array}\right]<0 .
$$

$$
\left[\begin{array}{ccc}
-2 P & P(A+\Delta A)-D+S & P\left(A^{\tau}+\Delta A^{\tau}\right) \\
(A+\Delta A)^{T} P-D+S & D(A+\Delta A)+(A+\Delta A)^{T} D+Q-2 S & D\left(A^{\tau}+\Delta A^{\tau}\right) \\
\left(A^{\tau}+\Delta A^{\tau}\right)^{T} P & \left(A^{\tau}+\Delta A^{\tau}\right)^{T} D & -Q
\end{array}\right]<0 .
$$$$
\left[\begin{array}{cccc}
D-D A-A^{T} D-\epsilon_{1} E^{T} E-\epsilon_{2}\left(E^{\tau}\right)^{T} E^{\tau} & -\left(A^{\tau}\right)^{T} D & 0 & D H \\
-D A^{\tau} & D & D H^{\tau} & 0 \\
0 & \left(H^{\tau}\right)^{T} D & \epsilon_{2} I & 0 \\
H^{T} D & 0 & 0 & \epsilon_{1} I
\end{array}\right]>0
$$ 
Proof: Suppose that there exist matrices $P>0, Q>0$ and a diagonal matrix $D>0$ such that the LMI in (27) holds, then it is easy to see (27) is equivalent to

$$
\left[\begin{array}{ccc}
-2 P & P A & P A^{\tau} \\
A^{T} P & D A+A^{T} D+Q-2 D & D A^{\tau} \\
\left(A^{\tau}\right)^{T} P & \left(A^{\tau}\right)^{T} D & -Q
\end{array}\right]<0 .
$$

Now, choose

$$
\tilde{P}=P, \quad \tilde{Q}=Q, \quad \tilde{D}=D, \quad \tilde{S}=D .
$$

Then, by (32), it is easy to see that $\tilde{P}>0, \tilde{Q}>0$ and diagonal matrices $\tilde{D}>0$ and $\tilde{S}>0$ in (33) satisfy (31). This completes the proof.

Theorem 7: Suppose that there exist a matrix $K>0$ and a diagonal matrix $P>0$ such that (28) and (29) hold, then there exist matrices $\tilde{P}>0, \tilde{Q}>0$ and two diagonal matrices $\tilde{D}>0$ and $\tilde{S}>0$ satisfying the LMI in (31).

Proof: Suppose that there exist a matrix $K>0$ and a diagonal matrix $P>0$ such that (28) and (29) hold, it can be seen that there exists a scalar $\epsilon>0$ such that

$$
P A+A^{T} P+K+\epsilon I<0 .
$$

Choose a matrix $\hat{P}>0$ satisfying

$$
\begin{aligned}
& -2 \hat{P}^{-1}+A^{\tau}\left(A^{\tau}\right)^{T}<0 \\
& -\epsilon I+\left[A^{T}+P A^{\tau}\left(A^{\tau}\right)^{T}\right]\left[2 \hat{P}^{-1}-A^{\tau}\left(A^{\tau}\right)^{T}\right]^{-1} \\
& \quad \times\left[A+A^{\tau}\left(A^{\tau}\right)^{T} P\right]<0 .
\end{aligned}
$$

Then, by (29), (35), (36), and (34), it can be seen that

$$
\left[\begin{array}{ccc}
-2 \hat{P}^{-1} & A & A^{\tau} \\
A^{T} & P A+A^{T} P-2 P+I & P A^{\tau} \\
\left(A^{\tau}\right)^{T} & \left(A^{\tau}\right)^{T} P & -I
\end{array}\right]<0 .
$$

Pre- and post-multiplying (37) by $\operatorname{diag}(\hat{P}, I, I)$, we obtain

$$
\left[\begin{array}{ccc}
-2 \hat{P} & \hat{P} A & \hat{P} A^{\tau} \\
A^{T} \hat{P} & P A+A^{T} P-2 P+I & P A^{\tau} \\
\left(A^{\tau}\right)^{T} \hat{P} & \left(A^{\tau}\right)^{T} P & -I
\end{array}\right]<0 .
$$

Now, choose

$$
\tilde{P}=\hat{P}, \quad \tilde{Q}=I, \quad \tilde{D}=P, \quad \tilde{S}=P .
$$

Then, by (38), it is easy to see that $\tilde{P}>0, \tilde{Q}>0$ and diagonal matrices $\tilde{D}>0$ and $\tilde{S}>0$ in (39) satisfy (31). This completes the proof.

Remark 2: Theorems 6 and 7 show that the asymptotic stability conditions for delayed cellular neural networks in [14] and [2] are special cases of Theorem 1 in this paper. Furthermore, we note that in [14], it was shown that the stability conditions in [1] and [11] are special cases of Theorem 2. Therefore, by Theorem 6, we have that the stability conditions in [1] and [11] are also special cases of Theorem 1 in this paper.

For the robust stability result in Theorem 2, we have the following theorem.

Theorem 8: Suppose that there exist a diagonal matrix $D>0$ and scalars $\epsilon_{1}>0, \epsilon_{2}>0$ such that the LMI in (30) holds, then there exist matrices $\tilde{P}>0, \tilde{Q}>0$, two diagonal matrices $\tilde{D}>0, \tilde{S}>0$, and scalars $\tilde{\epsilon}_{1}>0$ and $\tilde{\epsilon}_{2}>0$ satisfying the LMI in (24); that is (see (40), as shown at the bottom of the page).

Proof: Suppose that there exist a diagonal matrix $D>0$ and scalars $\epsilon_{1}>0, \epsilon_{2}>0$ such that the LMI in (30) holds, then, by Schur complements, it follows from (30) that

$$
\begin{aligned}
& \quad M \triangleq D-\epsilon_{2}^{-1} D H^{\tau}\left(H^{\tau}\right)^{T} D>0, \\
& \text { and } \\
& \qquad \begin{array}{l}
D A \\
\quad+A^{T} D-D+\epsilon_{1} E^{T} E+\epsilon_{2}\left(E^{\tau}\right)^{T} E^{\tau} \\
\\
+\epsilon_{1}^{-1} D H H^{T} D+\left(A^{\tau}\right)^{T} D M^{-1} D A^{\tau}<0 .
\end{array}
\end{aligned}
$$

By this, we can choose a scalar $\delta>0$ such that

$$
\begin{aligned}
& D A+A^{T} D-2 D+\epsilon_{1} E^{T} E+\epsilon_{2}\left(E^{\tau}\right)^{T} E^{\tau}+\epsilon_{1}^{-1} D H H^{T} D \\
& \quad+\left(A^{\tau}\right)^{T} D M^{-1} D A^{\tau}+\epsilon_{2}^{-1} D H^{\tau}\left(H^{\tau}\right)^{T} D+D A^{\tau} \\
& \quad \times\left[\left(A^{\tau}\right)^{T} D M^{-1} D A^{\tau}+\delta I\right]^{-1}\left(A^{\tau}\right)^{T} D+\delta I<0 .
\end{aligned}
$$

Set

$$
\tilde{Q}=\left(A^{\tau}\right)^{T} D M^{-1} D A^{\tau}+\epsilon_{2}\left(E^{\tau}\right)^{T} E^{\tau}+\delta I .
$$

Then, we can re-write (42) as

$$
\begin{aligned}
D A & +A^{T} D+\tilde{Q}-2 D+\epsilon_{1} E^{T} E+\epsilon_{1}^{-1} D H H^{T} D \\
& +\epsilon_{2}^{-1} D H^{\tau}\left(H^{\tau}\right)^{T} D+D A^{\tau}\left[\tilde{Q}-\epsilon_{2}\left(E^{\tau}\right)^{T} E^{\tau}\right]^{-1}\left(A^{\tau}\right)^{T} D<0
\end{aligned}
$$

which, by Schur complements, implies that there exists a matrix $P>0$ such that we have (44), as shown at the bottom of the page. Now choose

$$
\tilde{P}=P, \quad \tilde{D}=D, \quad \tilde{S}=D, \quad \tilde{\epsilon}_{1}=\epsilon_{1}, \quad \tilde{\epsilon}_{2}=\epsilon_{2} .
$$

Then, by (44), it is easy to see that a matrix $\tilde{Q}>0$ in (43) and $\tilde{P}>0$, diagonal matrices $\tilde{D}>0$ and $\tilde{S}>0$, scalars $\tilde{\epsilon}_{1}>0, \tilde{\epsilon}_{2}>0$ in (45) satisfy (40). This completes the proof.

Remark 3: By Theorem 8, it can be seen that the robust stability result for DCNN in [15] is a special case of Theorem 2 in this paper, which implies that the robust stability result in Theorem 2 in this paper is less conservative than that in [15].

$$
\left[\begin{array}{ccccc}
-2 \tilde{P} & \tilde{P} A-\tilde{D}+\tilde{S} & \tilde{P} A^{\tau} & \tilde{P} H & \tilde{P} H^{\tau} \\
A^{T} \tilde{P}-\tilde{D}+\tilde{S} & \tilde{D} A+A^{T} \tilde{D}+\tilde{Q}-2 \tilde{S}+\tilde{\epsilon}_{1} E^{T} E & \tilde{D} A^{\tau} & \tilde{D} H & \tilde{D} H^{\tau} \\
\left(A^{\tau}\right)^{T} \tilde{P} & \left(A^{\tau}\right)^{T} \tilde{D} & \tilde{\epsilon}_{2}\left(E^{\tau}\right)^{T} E^{\tau}-\tilde{Q} & 0 & 0 \\
H^{T} \tilde{P} & H^{T} \tilde{D} & 0 & -\tilde{\epsilon}_{1} I & 0 \\
\left(H^{\tau}\right)^{T} \tilde{P} & \left(H^{\tau}\right)^{T} \tilde{D} & 0 & 0 & -\tilde{\epsilon}_{2} I
\end{array}\right]<0 .
$$

$$
\left[\begin{array}{ccccc}
-2 P & P A & P A^{\tau} & P H & P H^{\tau} \\
A^{T} P & D A+A^{T} D+\tilde{Q}-2 S+\epsilon_{1} E^{T} E & D A^{\tau} & D H & D H^{\tau} \\
\left(A^{\tau}\right)^{T} P & \left(A^{\tau}\right)^{T} D & \epsilon_{2}\left(E^{\tau}\right)^{T} E^{\tau}-\tilde{Q} & 0 & 0 \\
H^{T} P & H^{T} D & 0 & -\epsilon_{1} I & 0 \\
\left(H^{\tau}\right)^{T} P & \left(H^{\tau}\right)^{T} D & 0 & 0 & -\epsilon_{2} I
\end{array}\right]<0 .
$$


Now, we provide a numerical example to show the reduced conservativeness of the result in Theorem 2 .

Example 1: Consider an uncertain DCNN in (1) with parameters as

$$
\begin{aligned}
A & =\left[\begin{array}{ccc}
0.1063 & -0.9370 & 0.3655 \\
-0.1589 & 0.2141 & 0.2889 \\
0.5475 & 0.4478 & 0.0202
\end{array}\right] \\
A^{\tau} & =\left[\begin{array}{ccc}
-0.2031 & 0.1132 & 0.0702 \\
-0.1707 & 0.0888 & -0.0355 \\
0.0767 & 0.4425 & -0.0944
\end{array}\right] \\
H & =\left[\begin{array}{c}
0.2887 \\
-0.1702 \\
0.1246
\end{array}\right], \quad H^{\tau}=\left[\begin{array}{c}
0.2397 \\
0.2823 \\
-0.2976
\end{array}\right] \\
E & =\left[\begin{array}{lll}
0.0212 & 0.0238 & -0.1008
\end{array}\right], \\
E^{\tau} & =\left[\begin{array}{lll}
-0.0742 & 0.1082 & -0.0131
\end{array}\right] .
\end{aligned}
$$

Then, it can be verified that the robust stability condition in Theorem 5 is not satisfied. Thus, the robust stability condition in [15] fails to conclude whether this DCNN is asymptotically stable or not. However, by resorting to the Matlab LMI Control Toolbox, we find that the LMI in (24) is feasible. Therefore, by Theorem 2, we have that the uncertain DCNN has a unique equilibrium point which is globally robustly asymptotically stable.

\section{CONCLUSION}

This paper has studied the problem of global robust stability analysis for DCNNs with norm-bounded parameter uncertainties. A sufficient condition ensuring the uniqueness and global asymptotic stability of the equilibrium point for a nominal DCNN has been proposed in terms of an LMI. Based on this, a robust stability condition has been developed. It has been theoretically established that both the stability and robust stability results obtained in this paper contain some existing ones in the literature as special cases. A numerical example has been provided to demonstrate the reduced conservativeness of the proposed results.

\section{REFERENCES}

[1] S. Arik, "An analysis of global asymptotic stability of delayed cellular neural networks," IEEE Trans. Neural Netw., vol. 13, no. 5, pp. 1239-1242, Sep. 2002.

[2] - "An improved global stability result for delayed cellular neural networks," IEEE Trans. Circuits Syst. I, vol. 49, no. 8, pp. 1211-1214, Aug. 2002

[3] S. Arik and V. Tavsanoglu, "On the global asymptotic stability of delayed cellular neural networks," IEEE Trans. Circuits Syst. I, vol. 47, no. 4, pp. 571-574, Apr. 2000.

[4] S. Boyd, L. El Ghaoui, E. Feron, and V. Balakrishnan, "Linear matrix inequalities in system and control theory," in SIAM Studies in Applied Mathematics. Philadelphia, PA: SIAM, 1994.

[5] J. Cao, "Global stability conditions for delayed CNN's," IEEE Trans. Circuits Syst. I, vol. 48, no. 11, pp. 1330-1333, Nov. 2001.

[6] J. Cao and T. Chen, "Globally exponentially robust stability and periodicity of delayed neural networks," Chaos, Solitons, \& Fractals, vol. 22, pp. 957-963, 2004.

[7] J. Cao, D.-S. Huang, and Y. Qu, "Global robust stability of delayed recurrent neural networks," Chaos, Solitons, \& Fractals, vol. 23, pp. 221-229, 2005.

[8] L. O. Chua and L. Yang, "Cellular neural networks: Applications," IEEE Trans. Circuits Syst., vol. 35, no. 10, pp. 1273-1290, Oct. 1988.

[9] - "Cellular neural networks: Theory," IEEE Trans. Circuits Syst., vol. 35 , no. 10, pp. $1257-1272$, Oct. 1988.

[10] V. B. Kolmanovskii and A. D. Myshkis, Introduction to the Theory and Applications of Functional Differential Equations. Dordrecht, The Netherlands: Kluwer, 1999.

[11] T.-L. Liao and F.-C. Wang, "Global stability of cellular neural networks with time delay," IEEE Trans. Neural Netw., vol. 11, no. 6, pp. 1481-1484, Nov. 2000.

[12] X. Liao and K. Wong, "Robust stability of interval bidirectional associative memory neural network with time delays," IEEE Trans. Syst. Man Cybern. B, vol. 34, no. 2, pp. 1142-1154, Apr. 2004.

[13] T. Roska, C. W. Wu, M. Balsi, and L. O. Chua, "Stability and dynamics of delay-type general and cellular neural networks," IEEE Trans. Circuits Syst. I, vol. 39, no. 6, pp. 487-490, Jun. 1992.

[14] V. Singh, "A generalized LMI-based approach to the global asymptotic stability of delayed cellular neural networks," IEEE Trans. Neural Netw., vol. 15, no. 1, pp. 223-225, Jan. 2004.

[15] V. Singh, "Robust stability of cellular neural networks with delay: Linear matrix inequality approach," Proc. Inst. Elect. Eng., Control Theory Appl., vol. 151, pp. 125-129, 2004.

[16] S. Xu, J. Lam, D. W. C. Ho, and Y. Zou, "Global robust exponential stability analysis for interval recurrent neural networks," Phys. Lett. A, vol. 325, pp. 124-133, 2004. 